\title{
Chemische Sensoren basierend auf metallorganischen Netzwerken
}

\author{
Johannes Körber, Rosemarie Dittrich, Yvonne Joseph, \\ Institut für Elektronik- und Sensormaterialien, TU Bergakademie Freiberg, Deutschland \\ Kontakt:Yvonne.joseph@esm.tu-freiberg.de \\ Schlagwörter: HKUST, MOF, Metal Organic Framework, Layer by layer deposition
}

\begin{abstract}
Zusammenfassung
Die Grundlage für unsere Untersuchungen war die Herstellung von lagenweise (layer-by-layer) abgeschiedenen Komponenten mittels Quarzmikrowage. Für die Experimente wurde der MOF HKUST-1 ausgewählt und die anorganische und die organische Komponente abwechselnd durch die Fluidzelle geleitet und so der MOF auf einem Schwingquarz mit Au-Oberfläche erzeugt. Die Charakterisierung der abgeschiedenen Schichten erfolgte mittels XRD, XPS und REM. Durch Variation der Konzentrationen der Lösungen und der Temperatur der Abscheidung konnten die Schichtdicke und die Schichtqualität variiert werden. Massensensitive Untersuchungen des beschichteten Schwingquarzes zeigten, dass die Schichten sensorisch aktiv sind.
\end{abstract}

\section{Einleitung}

Eine zentrale Aufgabe für fortgeschrittene chemische Sensoren ist die Entwicklung von chemosensitiven Schichten. Dabei spielen zwei Eigenschaften des chemosensitiven Materials eine besonders wichtige Rolle. Zum einen muss das Material stark porös sein, um eine optimale Sensitivität zu erreichen und zum anderen sollten die Sorptionseigenschaften anpassbar sein, um eine gute Selektivität des chemischen Sensors zu ermöglichen. Als weitere Voraussetzungen sollte der Sensor miniaturisierbar und einfach elektrisch auszulesen sein. Metallorganische Netzwerke (MOF- metal organic framework) bieten hohe Oberflächen, sind miniaturisierbar und in ihrer Zusammensetzung leicht zu variieren, was sie neben neben der Gasspeicherung auch für die chemische Sensorik interessant macht. Aufgrund des modularen Aufbaus dieser Verbindungen (bestehend aus einer anorganischen und einer organischen Komponente) kann eine Vielzahl von Strukturen realisiert werden, in denen durch geziel-te Auswahl der organischen Linkermoleküle die Porengröße gesteuert oder funktionelle Gruppen in die Porenwände eingebracht werden können. Die 3D-Struktur der MOF's ist durch die anorganische, sogenannte SBU (secondary building unit) gekennzeichnet.

\section{Experimente}

2.1 Herstellung der Selbstassemblierenden Monolage (SAM)

Die Goldsubstrate wurden 10 Minuten in einer Piranha Ätzlösung gereinigt und anschließend mit deionisierten Wasser gespült. Die eigentliche SAM-Lösung bestand aus einer 20 $\mu \mathrm{M}$ 16Mercaptohexadecansäure (MHDA) zu der 5 Vol\% Essigsäure hinzugegeben wurden [1]. Die Lösung wurde auf $70^{\circ} \mathrm{C}$ erwärmt und die gereinigten Goldsubstrate für mindestens $48 \mathrm{~h}$ darin eingelegt. Nach anschließendem Spülen mit deionisierten Wasser und Trocknen unter einem Argongasstrom wurden die Substrate in die Beschichtungszelle an der Quarzmikrowaage eingebaut.

\section{$2.2 \quad$ Schichtabscheidung}

Mittels E1-QCM D konnte der Aufbau der Multilagen aus Kupferacetat (Sigma-Aldrich 99\%-ig) und Trimesinsäure (Aldrich, 95\%-ig) verfolgt werden. Dazu erfolgte die Beschichtung auf einem gereinigten Schwingquarz, dessen Goldelektrode und mit SAM beschicht wurde. Die zwei Reaktionslösungen Kupferacetat und Trimesinsäure (Sigma Aldrich, Analysenqualität) wurden abwechselnd über die Substrate geleitet und zwischen- 
durch jeweils mit Ethanol gespült, um ungebundene Reste der Chemikalien von der Oberfläche zu entfernen.

Beschichtungszyklus:

1.Kupfer(II)-acetat $\left(\mathrm{Cu}_{2}(\mathrm{OAc})_{4}\right)$

2.Ethanol

\section{Trimesinsäure $\left(\mathrm{H}_{3} \mathrm{BTC}\right)$}

\section{Ethanol}

Die Abscheide- und Spülzeit betrug jeweils 8 Minuten und die Konzentration des Kupferacetat abhängig vom Experiment $0,1 \mathrm{mM} ; 0,5 \mathrm{mM}$ oder $1,0 \mathrm{mM}$, wobei die Trimesinsäure konstant bei $0,1 \mathrm{mM}$ gehalten wurde. Alle Substrate sind entweder untemperiert bei Raumtemperatur einge-setzt worden oder wurden auf $70^{\circ} \mathrm{C}$ erwärmt.

\section{$3 \quad$ Charakterisierung}

Mit der Quarzmikrowaage (QCM) wurde die Masseänderung auf dem Schwingquarz resultierend aus der Änderung der Schwingfrequenz während der Beschichtung bestimmt. Zur Untersuchung der Oberflächenmorphologie sowie für die Querschnittsaufnahmen der abgeschiedenen Schichten diente ein Rasterelektronenmikroskop (REM). Die Zusammensetzung des Kupfer-MOF's wurde qualitativ mittels Röntgen-PhotoelektronenSpektroskopie (XPS) nachgewiesen. Der Nachweis, dass HKUST-1 abgeschieden wurde, erfolgte durch Röntgendiffraktometrie (XRD) unter streifendem Einfall (Winkel $\sim 1^{\circ}$ ). Erste Sensorische Messungen an den HKUST-1-Schichten wurden mit feuchter Luft unter Verwendung eines Schwingquarzes durchgeführt.

\subsection{QCM-Messungen}

Die Masseänderung während der Schichtabscheidung ist direkt über die Sauerbrey-Gleichung mit der Änderung der Frequenz verbunden. Der Frequenzshift für eine typische Schichtabscheidung ist in Abb. 1 zu sehen.

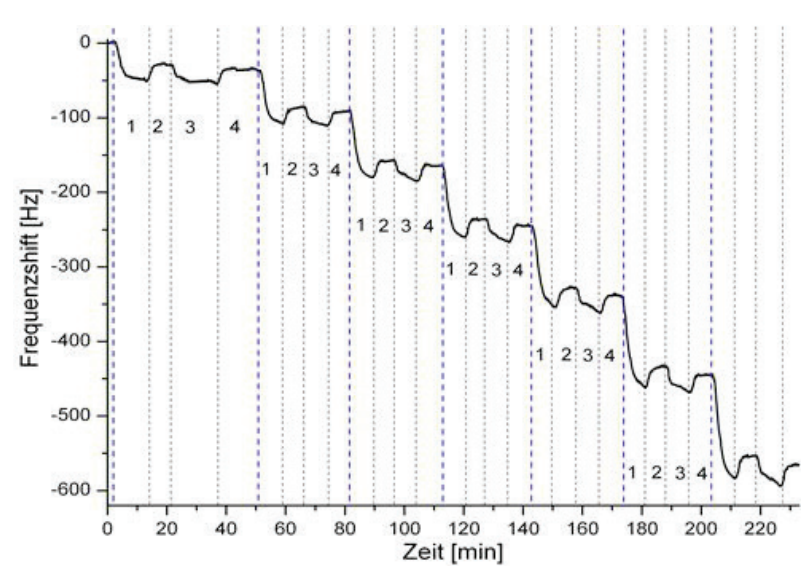

Abb.1: Frequenzshift während der Schichtabscheidung; 1. Kupferacetat (8min), 2. Ethanol (8min), 3. Trimesinsäure (8min), 4. Ethanol (8min); für den ersten Zyklus wurde eine längere Zeit für die Trimesinsäure und den anschließenden Spülschritt gewählt

In den ersten Beschichtungszyklen ist der Frequenzshift mit $<50 \mathrm{~Hz}$ und die damit verbundene Abscheidung noch gering. Nach dem "Einpendeln“ des Systems liegen saubere Schichten vor, auf denen sich die darauf folgenden Monolagen besser assemblieren. Der Frequenzshift beträgt hier rund $100 \mathrm{~Hz}$.

\subsection{REM-Untersuchungen}

Das Rasterelektronenmikroskop wurde eingesetzt um Aufnahmen der MOF-Oberfläche sowie Querschnittsuntersuchungen der Beschichtung anzufertigen. In Abb.2 ist die Oberflächenmorphologie für unterschiedliche Kupferacetatkonzentrationen und Abscheidetemperaturen zu sehen.

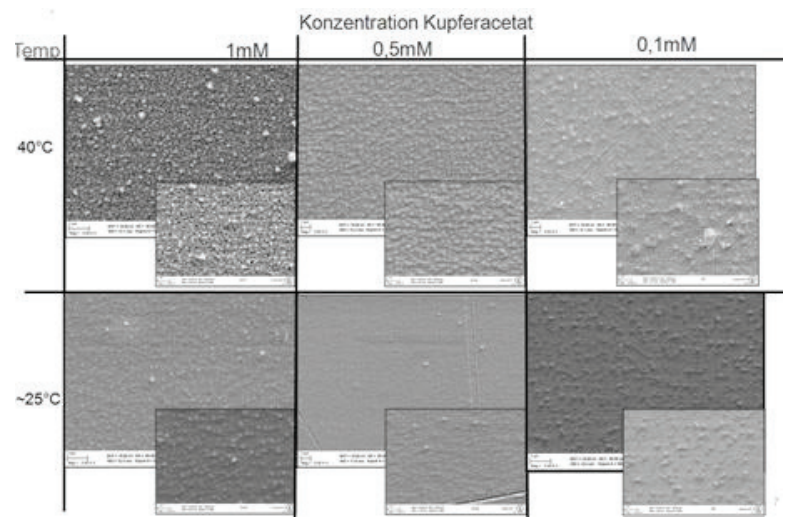

Abb.1: REM-Aufnahmen der Oberfläche für unterschiedliche Konzentrationen an Kupferacetat und verschiedene Abscheidetemperaturen

Auffällig ist, dass bei einer geringeren Kupferacetatkonzentration und Temperatur eine feinstruk- 
turiertere Oberfläche entsteht. Alle Oberflächen wurden mit 24 Beschichtungszyklen angefertigt.

Die Querschnittsaufnahmen sind in Abb.3 zu sehen.

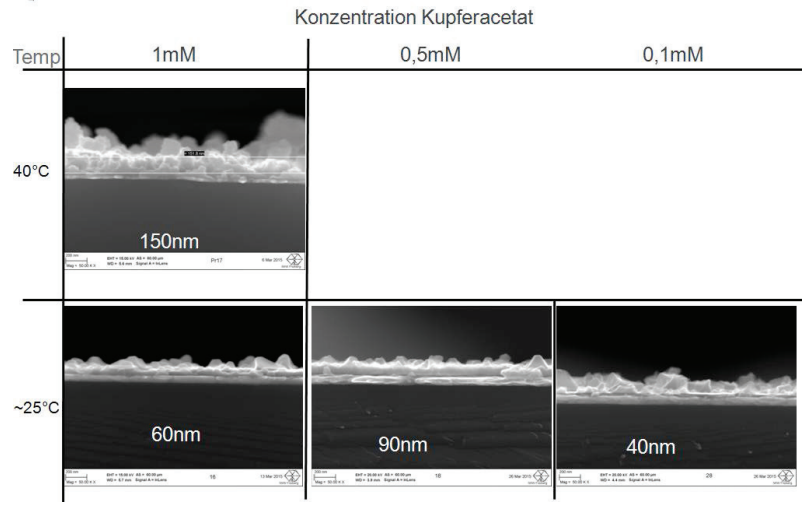

Abb.2: Querschnittsaufnahmen der HKUSTSchichten auf Si-Wafer (30 Beschichtungszyklen)

Für die Prozessparameter $1 \mathrm{mM}$ Kupferacetat und $40^{\circ} \mathrm{C}$ ist die mit Abstand höchste Schichtdicke zu verzeichnen $(150 \mathrm{~nm})$. Allerdings stammt diese Aufnahme auch von einem Schwingquarz als Substrat und ist mit den Si-Wafern bei $25^{\circ} \mathrm{C}$ nur bedingt vergleichbar. Niedrigere Konzentrationen ergeben geringere Schichtdicken. Erwartet sind laut Wöll für 30 Zyklen 50nm [2]. Daraus ergibt sich die Notwendigkeit, die Beschichtungszelle mit einer Heizung zu versehen, um die Anzahl der Beschichtungszyklen reduzieren zu können.

\subsection{XPS-Untersuchungen}

In Abb.4 ist die elementspezifische Zusammensetzung des HKUST-1 gezeigt.

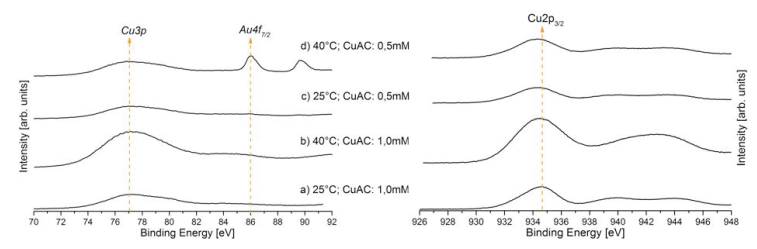

Abb. 3: XPS-Aufnahmen für Cu3p und Cu2 $p_{3 / 2}$

Nur für die $40^{\circ} \mathrm{C}$ und $0,5 \mathrm{mM}$ Kupferacetat Konzentration ist Gold sichtbar, für alle anderen Prozessparameter ist die MOF-Schicht geschlossen und das Gold Substrat nicht bestimmbar.

\subsection{XRD-Untersuchungen}

Die Zusammensetzung der abgeschiedenen MOFVerbindung wurde mittels XRD unter streifendem Einfall ermittelt. Neben dem Gold als Elektrodenmaterial konnten im Bereich $10-20^{\circ}$ (2 theta) die für den HKUST-1 charakteristischen Peaks nachgewiesen werden.



Abb.4: Röntgendiffraktogramm der abgeschiedenen Schicht

\subsection{Sensorische Untersuchungen}

Für die sensorischen Messungen wurde auf einen Schwingquarz eine HKUST-Schicht aufgebracht [3]. Über ein geschlossenes System gelangte Luft, die über einen Sättiger mit 3600ppm Wasser gemischt wurde, an die HKUST-Oberfläche. Die Untersuchungen erfolgten an temperatur- und konzentrationsabhängig abgeschiedenen Schichten. Gemessen wurde die Änderung der Schwingfrequenz des Quarzes.

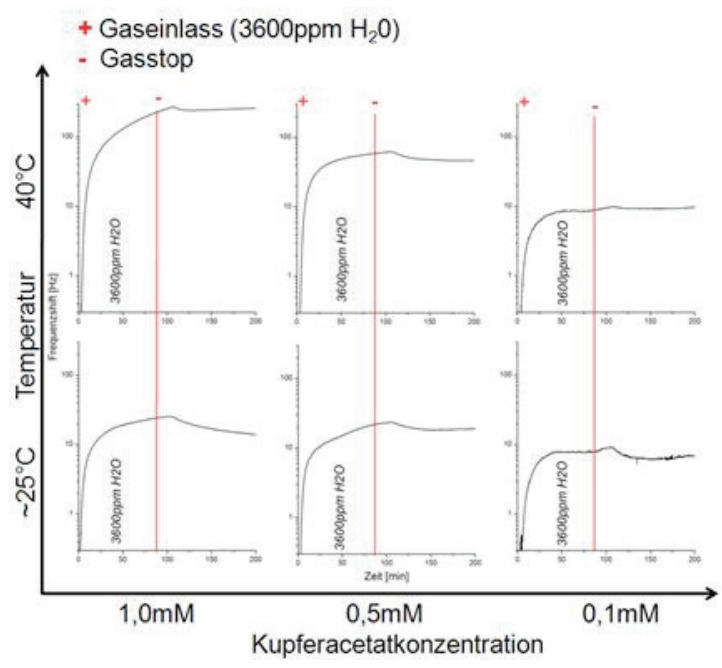

Abb.6: Wasserdampfadsorptionsmessungen

Sowohl eine Beschichtung bei höherer Temperatur als auch bei höherer Kupferacetatkonzentration 
ergibt eine Zunahme Wasserdampfadsorption (siehe Abb.6). Die Messzelle besaß ein großes Totvolumen (1I), womit die langen Ansprechzeiten bei den sensorischen Messungen erklärbar sind. Die Messzelle sollte für nachfolgende Untersuchungen stark verkleinert werden und mit einer Heizung zur Unterstützung der Desorption der Analyte ausgestattet werden.

\section{Literature}

[1] Wang, H.: Facile route for synthesis and controlled growth of SURMOFs on functionaliced organic surfaces.

[2] Dissertation Ruhr-Universität Bochum, 2010 metalorganic frameworks Materialstoday März 2012 (15)3

[3] Biemmi, E. ; Darga, A.; Stock N.; Bein, T.: Direct growth of $\mathrm{Cu} 3(\mathrm{BTC}) 2(\mathrm{H} 2 \mathrm{O}) 3 \times \mathrm{H} 2 \mathrm{O}$ thin films on modified QCM-gold electrodes - Water sorption isotherm.

Microporous and Mesoporous Materials 114 (2008) 380-386

\section{Danksagung}

Wir danken den Instituten für Experimentelle Physik und für Werkstoffwissenschaft der TU Bergakademie Freiberg für die Unterstützung bei den XPS-Messungen und den REM-und XRD-Untersuchungen. 